\title{
'Explorances' or Why (Some) Physical Entities Help Us Be More Creative
}

\author{
Sowmya Somanath, Ehud Sharlin, Mario Costa Sousa* \\ University of Calgary, Calgary, Canada
}

\section{Introduction}

We believe that every physical entity has a set of attributes that defines the degree of how creatively it can be used. For example, the affordances, abstractness and modular nature of Lego ${ }^{T M}$ blocks allows them to take on different forms of expression that showcases varying levels of human creativity (e.g. building alphabets, creating homes etc.). Similarly, when a DIY designer uses bottles to build houses, it projects his creative skills, but at the same time it speaks about the materiality, affordances and embodiment of the bottle which lends itself readily to creative and novel interaction design efforts. This theory, that every entity has a set of attributes that allows them to lend themselves more or less readily to creative and novel interactive design explorations is what we call explorances and is the focus of our proposed work.

Explorances is defined as an attribute of an entity that allows it to be used in a manner that defies former dogmas, explored in ways that support breaking free from its existing regular definition, form and context, to support novel and creative design efforts. In other words, explorances can be looked upon as a theoretical lens that will allow people to practice and/or analyze creative and interactive design efforts. Although the ability to create something novel stems out of a person's mind, the creative process is supported, facilitated and analyzed by a diverse range of disciplines, such as psychology, design, material science, technology, arts etc. For example, designers have long known that the properties of different materials can influence creativity i.e., as part of their common practice, designers engage in design explorations where they focus explicitly and exhaustively on the properties of one material or a set of materials. However, understanding what this designer's appropriation is challenging and what we call explorances. Our research goal is to better identify what those entity attributes are and incorporate them within user interface design. In the future we would also like to explore how we can quantify explorances to compare two or more entities in terms of their ability to better facilitate creativity in design work-flow processes.

\section{Our Approach}

In our current approach we apply our theory to the design of a tangible user interface (TUI) [Ishii and Ullmer 1997]. Doodling Wells is a TUI designed to assist in the process of inputting preliminary $3 \mathrm{D}$ curves using direct input method. The application context for this TUI is the domain of oil-and-gas engineering and the 3D curve is representative of the oil wells that are bored into the earth to extract hydrocarbons (i.e. oil) from underground oil/gas reservoirs.

The straightforward benefit of our TUI prototype is the physical feedback that it is provides to the reservoir engineer as they are designing the well trajectory, unlike other traditional approaches such as desktop based solutions, or virtual reality (VR) approaches currently being explored by the domain. While the system in its current form lacks preciseness needed for meticulous well planning tasks, the system is beneficial for quick and easy design prototyping.

The Doodling Wells prototype is based on the metaphor of sketching, and uses a handheld 3D printer, 3 Doodler $^{T M}$. The advantage of using the $3 D^{2}$ oodler ${ }^{T M}$ is that it provides greater flexibility in

*e-mail:\{ssomanat,ehud,smcosta $\} @$ ucalgary.ca

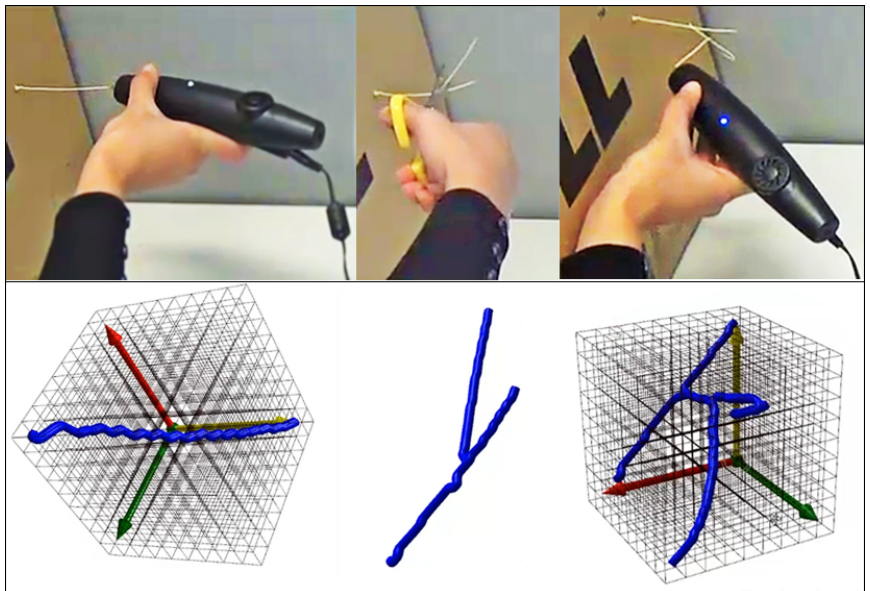

Figure 1: Doodling Wells: designing and revising well trajectories.

terms of sketching free-form curves compared to molding or manipulating other physical materials like clay, metal wire etc. Since the act of inputting curves is dynamic, we cannot track by tagging the physical objects. Thus, we chose to use Kinect 2.0 to perform tag-less tracking of the physical objects. The improved depth camera resolution of the new Kinect allows to better track and approximate thin objects like the plastic output from the 3 Doodler ${ }^{T M}$. A single Kinect is being used in this experiment, and hence we only capture the visible face of the tracked object. The Kinect is placed at an angle to capture maximum information about the shape of the curve. The skeleton of the curve is extracted and to get a relatively smooth curve that approximates the input curve in 3D we apply the Chaikin algorithm. Figure 1 demonstrates inputting a physical well trajectory using the 3 Doodler ${ }^{T M}$. The engineer in this sequence starts with a simple physical horizontal well configuration, which is digitally reconstructed. To revise the curve the engineer can use an additive style physical modeling wherein we add new features to an existing structure or could easily break the curve as shown in the Figure 1 to start afresh.

The key attributes that we think contribute towards the explorances for this TUI are: (a) modularity, i.e. the ability to remove or add to the curve quickly and easily; (b) abstraction and embodiment, i.e. the representation of the curve could be interpreted differently based on task context (e.g. well inputting, concept modeling) and environment (e.g. art studio vs classroom) and lastly, (c) affordances of the material to lend itself to the activity of sketching.

In our future work, we would like to test our system in practice to confirm the viability of this approach. We also want to explore other work groups, i.e. concept artists, to better learn about the effects of creativity on the work process and incorporate those design attributes into user interface design.

\section{References}

ISHII, H., AND Ullmer, B. 1997. Tangible bits: towards seamless interfaces between people, bits and atoms. In Proc. of the SIGCHI, ACM, 234-241. 